

\section{Microcontroller Based Self-Regulating Devices in Enclosed Environments}

\section{Shalaka Subrahmanyam1, Varun Panicker ${ }^{2}$}

${ }^{1}$ Professor, Thadomal Shahani Engineering College, India ${ }^{2}$ Professor, Thadomal Shahani Engineering College, India 


\section{Microcontroller Based Self-Regulating Devices in Enclosed Environments}

ASTRACT - Recent times have witnessed developments in the integration of wireless
communication and sensing, along with electronic programmable controller devices in real-time operating systems. Intelligent devices incorporate ambient intelligence into enclosed environments by compiling the information received by wireless sensors and providing necessary feedback. This paper analyses the ubiquitous devices with smart sensing technology by casting its assimilation, thereby modelling the actualization of an intelligent environment. Further, we propose a prototype environment using adept embedded system based devices.

\section{KEYWORDS - 8051, AMBIENT INTELLIGENCE, ATMEL AT89C52, AUTOMATION, AUTOMATED LIGHT, DC SUPPLY, RELAY}

\section{INTRODUCTION}

The human race has leaped towards advancement in the past century. These technological advancements have found a way into our homes, and the inclusion of such innovations into our lives has majorly improved our standard of living and has made living life a lot easier. A self-regulating device is a device that collects data and processes it, eventually bringing about changes which would otherwise require human intervention. This technology has gained immense popularity in recent years owing to the convenience with which it can be implemented and the benefits that it offers. Self-regulatory devices enjoy preference over manual systems as they conserve a very good amount of energy. These devices can be controlled by different methods; such as a remote control, an application on a mobile phone, Bluetooth, gestures, voice, internet etc. It can operate for devices like centralized HVAC (heating, ventilation, and air conditioning), lighting, security systems, garage doors, water irrigation and other such appliances. This is a great advantage for the elderly and the physically handicapped. The ease with which it can be operated makes it a very viable option for them as well. 


\section{NEED FOR AUTOMATION}

1. It can replace human mediation, and produce results faster

2. The results obtained are computerized and accurate, and are thus less prone to error

3. It is a power saving alternative

4. As there is low human involvement in the working of these devices, it is convenient

5. It is very cost- effective in the long run

6. It can perform tasks which would otherwise be dangerous for humans

\section{HARDWARE}

\section{SUPPLY}

The power supply available for residential and commercial applications is usually $230 \mathrm{~V}$, but the voltage we require to power a microcontroller circuit is approximately $5 \mathrm{~V}$. It is therefore necessary to build a power supply unit for the specified application.
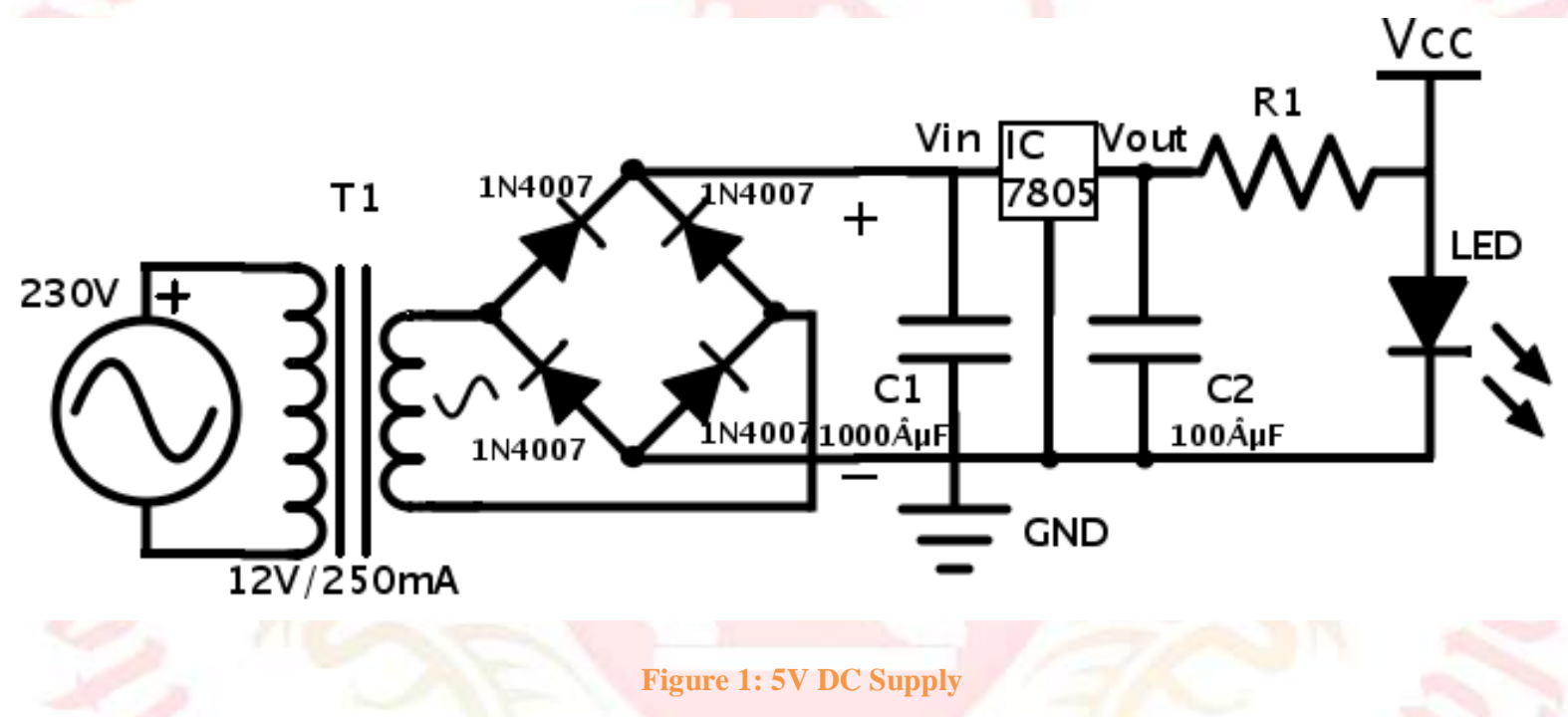

The circuit as in Figure-1 shows the DC supply unit. AC supply is given to the circuit through a step down transformer. The maximum output power of the supply unit is calculated as $\mathrm{P}=\mathrm{V}^{*} \mathrm{I}$; where $\mathrm{V}$ and $\mathrm{I}$ are the voltage rating and current rating respectively of the transformer. In this project a $12 \mathrm{~V} / 250 \mathrm{~mA}$ transformer is used. This implies that the maximum output power of the supply unit will be $4 \mathrm{~W}$. The four diodes (1N4007) act as a full wave rectifier. The output of the rectifier is a pulsed DC supply. Capacitor $\mathrm{C} 1(100 \mu \mathrm{F} / 25 \mathrm{~V})$ removes the AC ripples present in the acquired DC supply to make it pure DC. IC7805 is a regulator IC used to regulate the input voltage. Even though the input voltage fluctuates, the output voltage stays fixed. Thus a constant $5 \mathrm{~V}$ supply from $12 \mathrm{~V}$ DC is obtained to drive the circuit. Capacitor $\mathrm{C} 2$ $(100 \mu \mathrm{F} / 50 \mathrm{~V})$ gets rid of any unwanted ripple in the output. An LED is simply used to confirm that the circuit is ON. This circuit has been used to power the microcontroller and relay units. 


\section{CONTROL UNIT- ATMEL AT89C52}

The Atmel AT89C52 is a CMOS 8-bit microcontroller which uses low power and has high performance. It is 8051-based and consists of $32 \mathrm{I} / \mathrm{O}$ Lines, 3 Timers/Counters each of 16 bits, 8 Interrupt sources/2 Priority Levels, UART, Three-Level Program Memory Lock, 8K Bytes each Programmable and Erasable Read Only Memory(PEROM) and Flash memory as well as 256 Bytes RAM. It is a CMOS controller which fully static and has a clock frequency of $24 \mathrm{MHz}$. It can withstand upto $1000 \mathrm{Write} /$ Erase cycles implying that it can be erased and programmed to a maximum of 1000 times. It is consistent with the industry typical 80C51 and 80C52 instruction set and pinout. Atmel AT89C52 is a highly flexible and cost-effective solution for various embedded system applications as it combines a versatile 8 bit CPU with Flash memory on a monolithic chip.

\section{TO V CONVERTER}

The application of 'Indoor self- regulating plant watering system' requires a comparison of threshold voltage to voltage between two electrodes. Thus, a current-to-voltage converter, which is simply a shunt resistor, is connected before the comparator to convert the current flowing between the two electrodes to a voltage value.

\section{OPERATIONAL AMPLIFIER}

An op-amp(IC 741) is used as a comparator to compare threshold voltage to voltage between the electrodes. There are two inputs to the comparator viz. V+ and V-. The output is taken through Vo.

$\mathrm{Vo}=\mathrm{HIGH}$, when $\mathrm{V}+>\mathrm{V}-$

$\mathrm{Vo}=\mathrm{LOW}$, when $\mathrm{V}+<\mathrm{V}-$

\section{UP/DOWN BI-DIRECTIONAL COUNTER}

Up/down Bi-directional counter performs the function of counting in both directions. A counter which can count values from 0 to 9999 is used. The counter increments by 1 in up-state and decrements by 1 in down-state.

\section{RELAY CIRCUIT}

A relay circuit usually is a smaller switch like device that is used to open or close an electric switch efficient enough to carry higher currents to drive a higher current requirement device.

\section{NAND Gate}

A NAND gate output is HIGH only when all the inputs to the gate are LOW. In this paper, a two-input NAND gate is used. The output of this gate is HIGH when both the inputs are LOW and the output is LOW when at least one input is HIGH. 


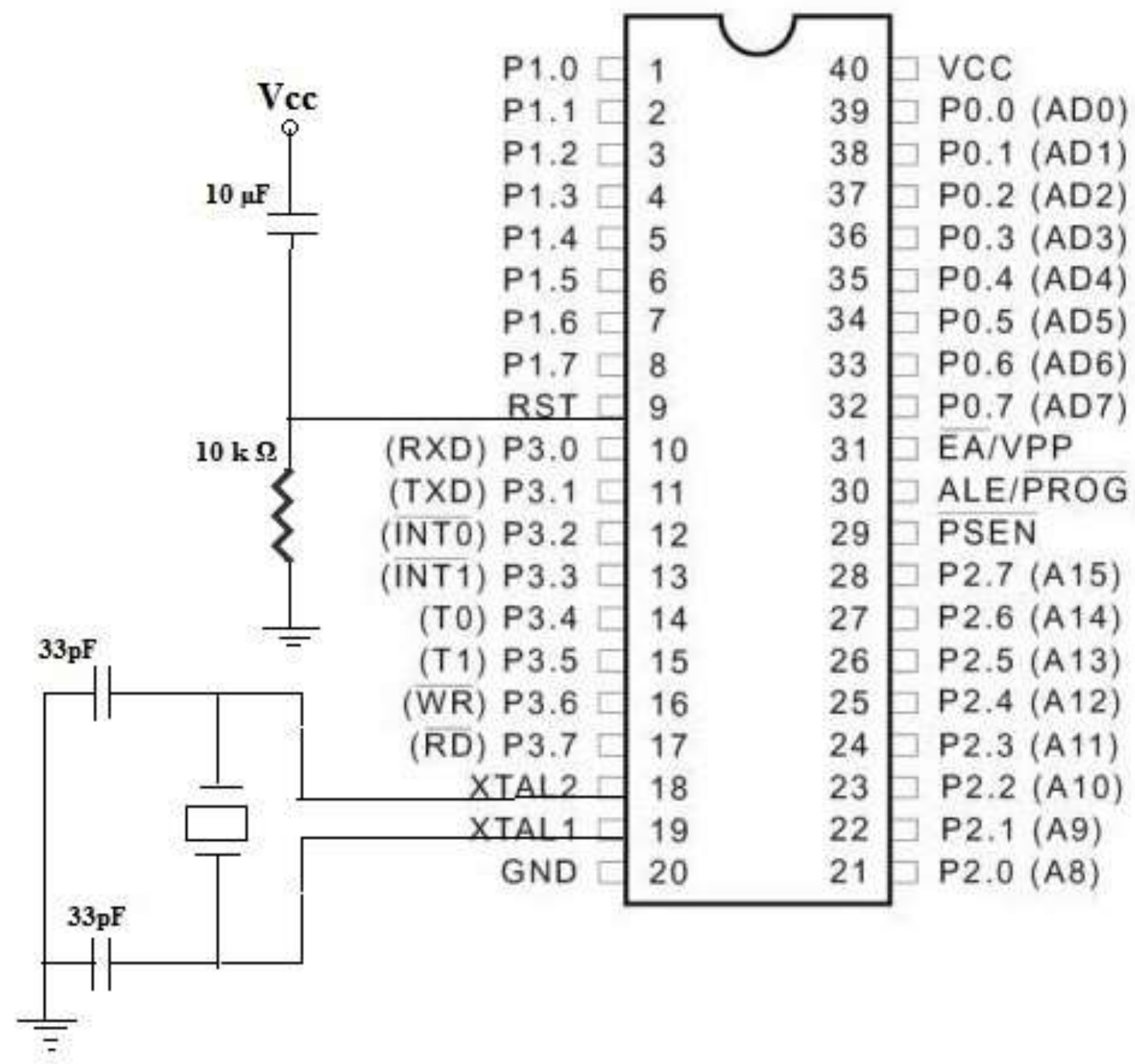

Figure 2: AT89C52 Pin Diagram

\section{SOFTWARE}

Embedded $\mathrm{C}$ programming is the assembly language embedded into the Microcontroller used for this project.

\section{APPLICATIONS}

\section{BI-DIRECTIONAL COUNTER DEPENDENT AUTOMATED LIGHT SWITCHING SYSTEM}

An up down counter which can change its selector state in both directions, and the direction of counting controlled by an up down selector input, is used to count the number of individuals entering or leaving the gate. The selector controller is connected to a turnstile. When the turnstile is in clockwise direction, the selector is in the up state, indicating the entry of an individual, thus incrementing the counter. Likewise, 
when the turnstile is in the anti-clockwise direction, the selector is in the down state, indicating the exit of an individual, thereby decrementing the counter. Once the last individual exits, the counter goes to zero, single pole dabble throw relay connected to the microcontroller turns off, light turns off. $12 \mathrm{~V} / 100 \mathrm{~mA}$ is required to operate the relay, which cannot be supplied by the microcontroller. Thus a driver transistor is added between the microcontroller and the SPDT. This is an effective power saving method.

Table 1: Truth Table NAND Gate

\begin{tabular}{|l|l|l|}
\hline X Input & Y Input & Output \\
\hline $\mathbf{0}$ & 0 & 1 \\
\hline $\mathbf{0}$ & 1 & 0 \\
\hline $\mathbf{1}$ & 0 & 0 \\
\hline $\mathbf{1}$ & 1 & 1 \\
\hline
\end{tabular}

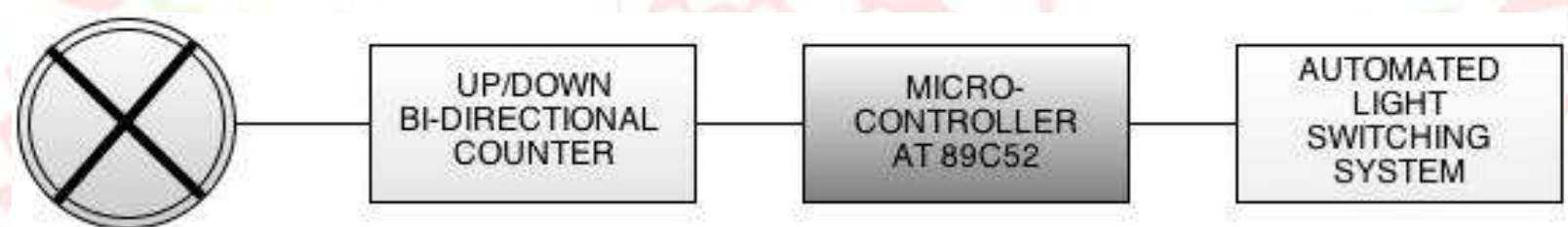

\section{TURNSTILE}

Figure 3: Block Diagram- Bi-Directional Counter Dependent Automated Light Switching System

\section{ALGORITHM}

1. Initialize the counter to zero

2. Define the input and output ports of the microcontroller

3. Interface the counter with the turnstile

4. If the turnstile rotates in the clockwise direction, increment counter by 1

5. Else if the turnstile rotates in the anti-clockwise direction, decrement the counter by 1

6. Embed assembly program into microcontroller

7. Decision box- Counter value $==0$ ?

- Yes: Relay OFF

- No : Relay ON

Relay $\mathrm{OFF}=>$ Light $\mathrm{OFF}$

Relay ON $=>$ Light ON

Repeat steps 4 to 8 in an infinite loop 


\section{PROGRAMMED INTELLIGENT WATER OVERFLOW CONTROLLER}

An overflow controller operates on the principle of conduction of electricity by water. It prevents the wastage of water and therefore finds use in various places where it is important to avoid the spillage of water such as office or home water tanks. A NAND gate is used between the microcontroller probe and the relay circuit. The other input to the NAND gate is Vcc (Binary 1). The output of the NAND gate controls the relay circuit, further; the output of the relay circuit drives the switching circuit of the water tap system. In the case that the water is in contact with the probe of the microcontroller, conduction of electricity takes place and therefore the output of the NAND gate is low. This will result in the relay being switched OFF, thus switching the water tap system OFF, thereby preventing overflow.

\section{ALGORITHM:}

1. Define the input and output ports of the microcontroller

2. Set water tap system ON

3. Embed assembly program into microcontroller

4. While NAND output $=\mathrm{HIGH}$, Relay $\mathrm{ON} \Rightarrow>$ Water tap system $\mathrm{ON}$

5. $\mathrm{NAND}$ output $=\mathrm{LOW}$, Relay $\mathrm{OFF}=>$ Water tap system OFF

6. Repeat steps 2 to 4 in an infinite loop
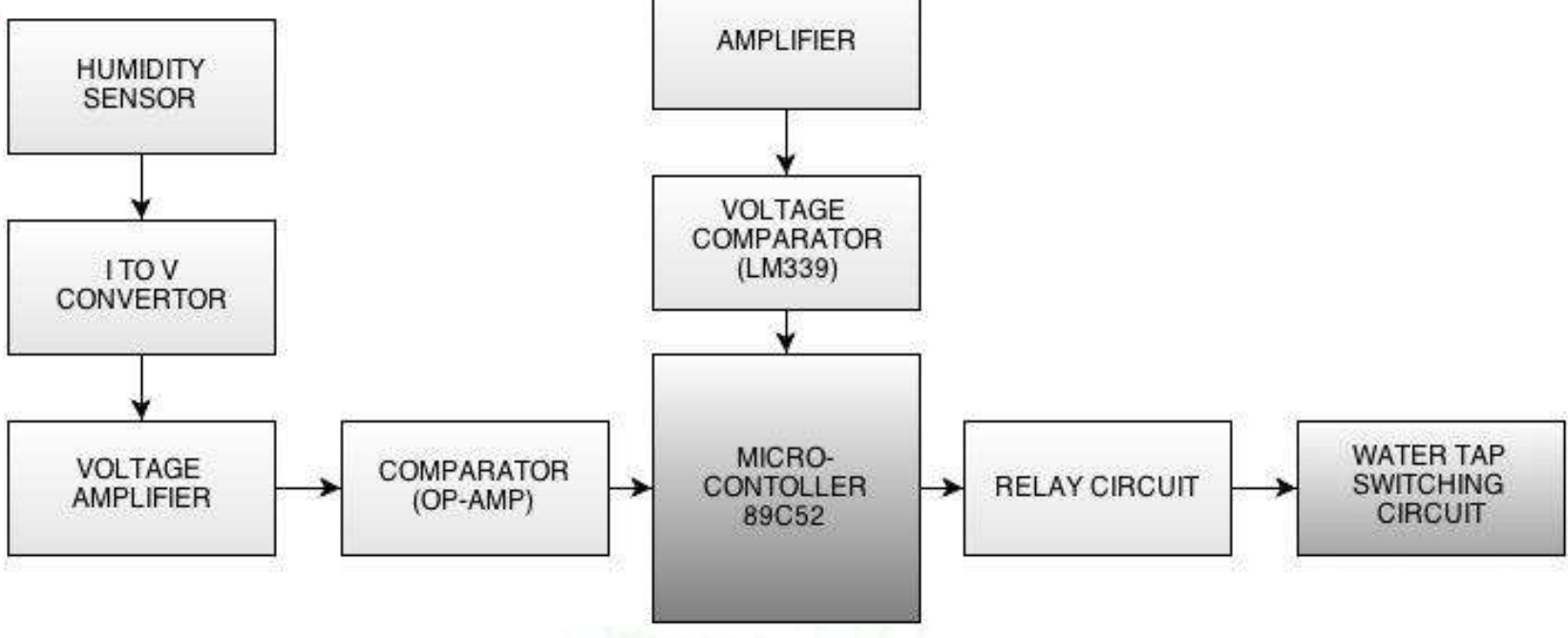

Figure 4: Block diagram- Programmed Intelligent Water Overflow controller 


\section{INDOOR SELF REGULATING PLANT WATERING SYSTEM}

Lately, it has become increasingly challenging for working individuals to maintain plants indoors. Also, due to scarcity of water in many regions it has become imperative to employ new techniques to save water. A self regulating water system enables us to check the humidity levels of soil and supply water directly to the plant when the moisture level of the soil is below a certain threshold level, pre-specified as the moisture level that is optimum for sustainable plant growth. Two metal electrodes are inserted into the soil. The resistivity of the soil is determined by the moisture content of the soil. When the moisture content is high, the resistivity of the soil is low, and less current is conducted leading to a lower voltage at the output of the I to $\mathrm{V}$ converter. The moisture content of the soil is inversely proportional to the current across the electrodes and consequently the voltage at the output of the I to $\mathrm{V}$ converter. The program is embedded into the microcontroller to check the moisture level and output of comparator which compares the threshold voltage to the amplified available voltage obtained as the voltage between the two electrodes. C program embedded into the microcontroller starts the tap when level is low, shuts the tap as soon as level reaches threshold and stays off till the level is low again.

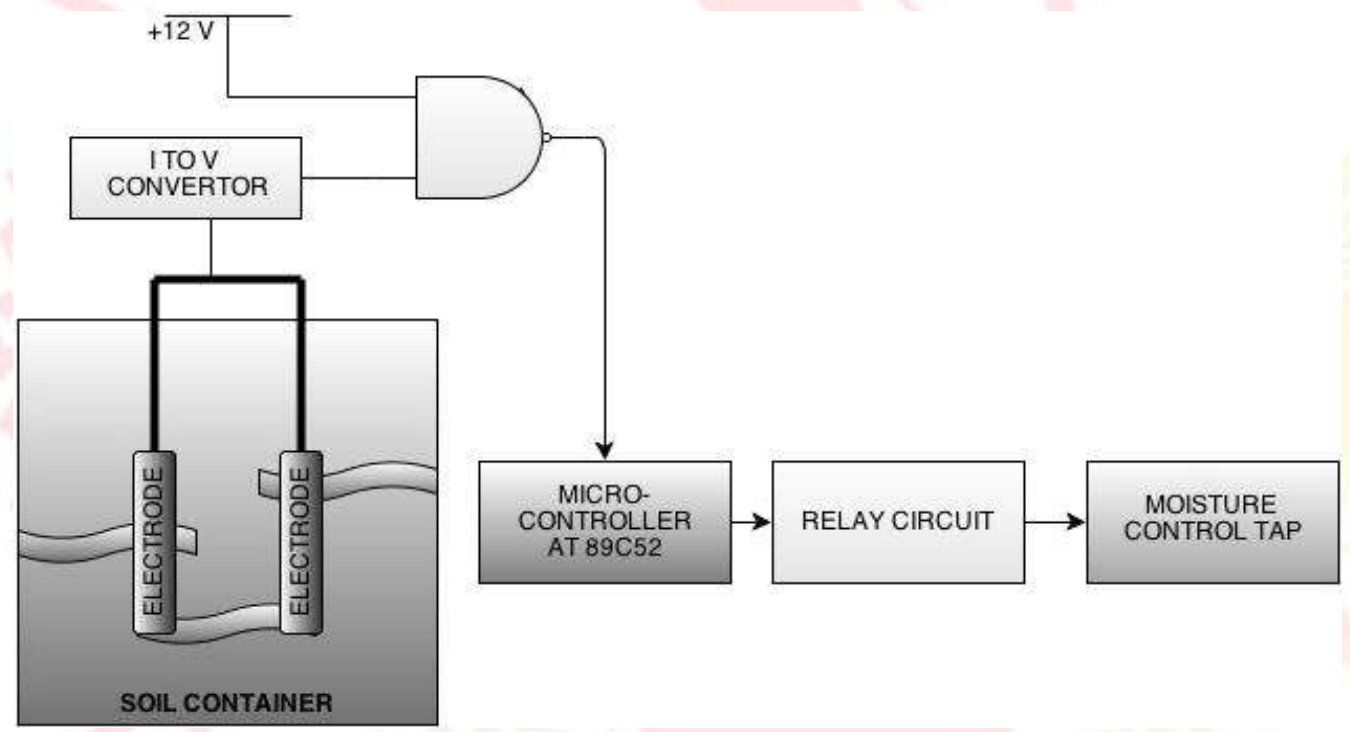

Figure 5: Block Diagram- Indoor Self Regulating Plant Watering System

\section{ALGORITHM:}

1. Define the input and output ports of the microcontroller

2. Embed assembly program into the microcontroller

3. Define the threshold voltage level relative to humidity required as $\mathrm{V}+$

4. Voltage between electrodes through the I to V converter is V-

5. Interface the humidity sensing circuit with the microcontroller

6. If $\mathrm{Vo}=\mathrm{HIGH}$, then level $=\mathrm{LOW} \Rightarrow$ Tap $\mathrm{ON}$

7. Else if $\mathrm{Vo}=\mathrm{LOW}$, then level $=\mathrm{HIGH}=>$ Tap OFF

8. Repeat steps 5 and 6 in an infinite loop 


\section{FUTURE PROSPECTS AND SCOPE}

The proposed project has a very vast scope in many areas, both, commercial as well as residential. Selfregulating devices can be set up in apartments as well as in office spaces. Though the initial cost of set-up is to be borne by the individual, in the long-run it proves to be a very lucrative alternative to manually-run devices. A prototype enclosed real-time environment would include self-regulating fans/air-conditioners and lights that adapt to the temperature and natural lighting automatically, without manual adjustments. In such a scenario, the lights will automatically switch on at dusk and switch off at dawn with the help of light sensors (LDR), and an air-conditioner will automatically regulate itself to a comfortable temperature depending on the ambient temperature using temperature sensors. Along with this, every device in a household can be automated in a similar manner. Such a smart prototype environment is the future prospect of this project. In the future, every device will be self-regulatory. Every single device will be controlled by a small chip which is the Microcontroller. Devices will be capable of being driven via the long-distance communication techniques such as Bluetooth, Infrared, Mobile telephones etc. Automation can also be used on a larger scale for smart applications like load-shedding, irrigation for agriculture, power consumption data interpretation in real-time to allow consumers to know exactly how much power is being used by each device etc.

\section{CONCLUSION}

This paper shows how the Atmel AT89C52 microcontroller can be interfaced with various systems and are capable of real time data processing and feedback. The implementation can be either extremely complex wherein every device connected to the power supply is automated, or it can be a simple automation of a single device. These systems' applications have been demonstrated in home automation. However, at sites where manual systems are already in place, it becomes cumbersome and slightly expensive to switch to an automated counterpart. Concepts such as the use of a turnstile to control the input to the counter and a NAND gate to control the input to the relay have been introduced. The block diagrams have been specifically used to model the hardware system with the underlying necessary details. The proposed models significantly improve reliability on implementation.

\section{REFERENCES}

[1]. "SMART HOME KNOWS JUST HOW YOU LIKE YOUR BREAKFAST". NewScientist: September 2009

[2]. Mark Edward Soper (2004). "ABSOLUTE BEGINNER'S GUIDE TO HOME AUTOMATION".

[3]. US Patent: EP1260886 A2, Claudio Bonassi, Pierangelo Colombo,Alberto Federico Giua, Michael Hauptman Robert N Bucceri (2003). "LATEST TECHNOLOGY IN AUTOMATED HOME CONTROL".

[4]. William C. Mann (ed.) (2005). "SMART TECHNOLOGY FOR AGING, DISABILITY AND INDEPENDENCE : THE STATE OF THE SCIENCE"; John Wiley and Sons

[5]. Jivan S. Parab et al.(2008). "PRACTICAL ASPECTS OF EMBEDDED SYSTEMS USING MICROCONTROLLERS" ; Springer

- END - 


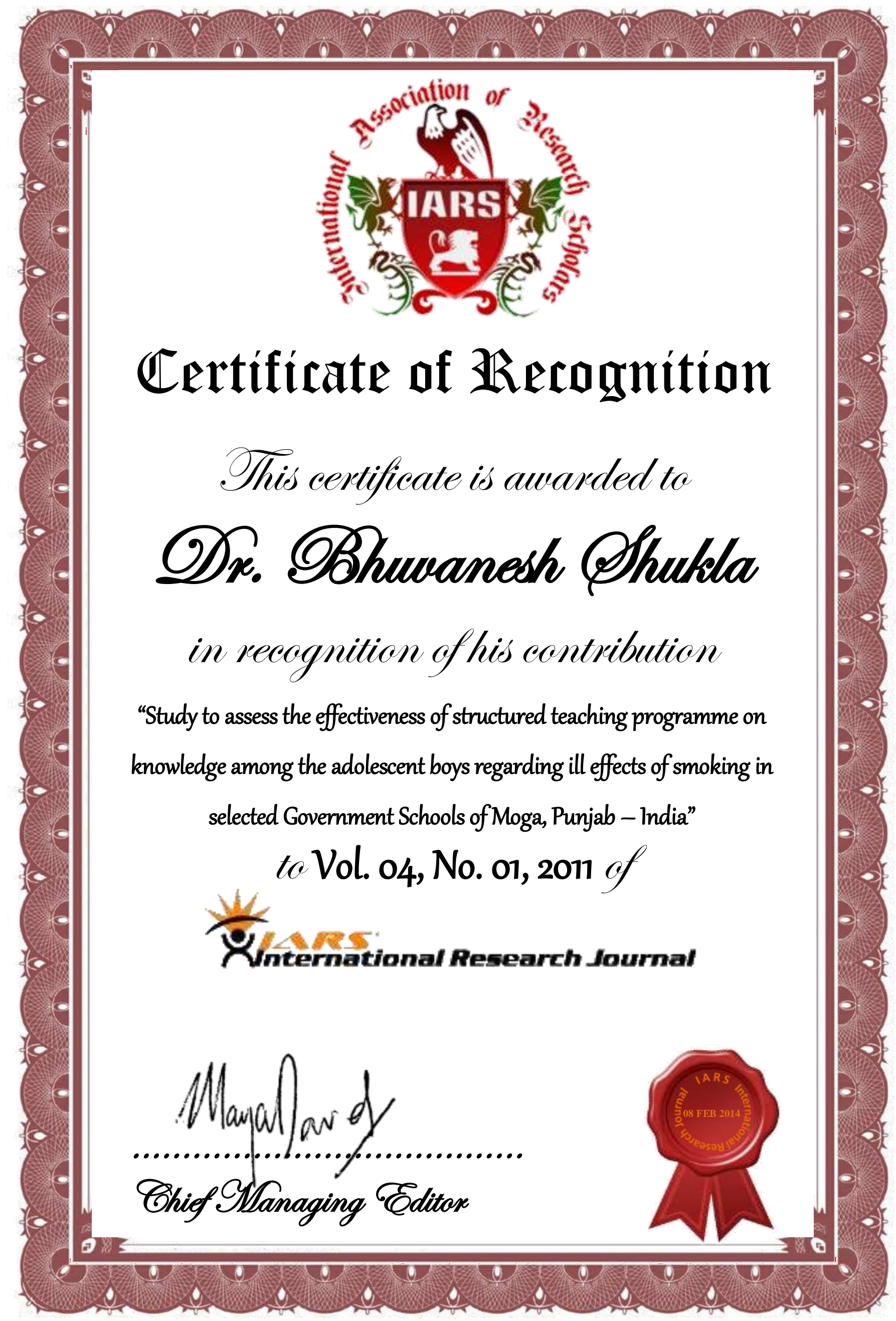

